\title{
Unusual Presentation of Metastatic Carcinoma of Prostate with Cervical Lymphadenopathy as Initial Presentation -A Case Report and Review
}

\author{
Authors \\ Zeba Chaudhary ${ }^{1}$, Shahid Anjum², Mohit Thalquotra ${ }^{3}$, Mudassir Khan ${ }^{4}$, \\ Abdul Hakim ${ }^{5 *}$ \\ ${ }^{1}$ Consultant Pathology Zara Labs, Rajouri, J\&K. \\ ${ }^{2}$ Consultant Surgery Health Department, J\&K. \\ ${ }^{3}$ Assistant Professor Biochemistry, GMC RAJOURI, J\&K. \\ ${ }^{4}$ Assistant Professor Surgery, GMC Rajouri, J\&K. \\ ${ }^{5}$ Assistant Professor Radiation Oncology, GMC Rajouri, J\&K. \\ *Corresponding author \\ Dr Abdul Hakim \\ Assistant Professor Radiation Oncology, GMC Rajouri, J\&K., India
}

\begin{abstract}
It is estimated that there are almost 1.3 million new cases of prostate cancer, ranking as the second most frequent cancer and the fifth leading cause of cancer death in men. It is the most frequently diagnosed cancer among men in over one-half (105 of 185) of the countries of the world.

Prostate cancer presenting as cervical lymphadenopathy as initial presentation is rare while most present with genitourinary symptoms. We present a case of a 97 years-old male who presented with supraclavicular lymphadenopathy as the primary complaint. FNAC from lymph node revealed metastatic adenocarcinoma. CECT chest and abdomen revealed large left cervical lymphadenopathy with few subcentimetric pre and paraaortic lymph nodes without any other obvious lesion. Serum PSA was grossly elevated with value $269 \mathrm{ng} / \mathrm{ml}$, based on PSA value diagnosis of metastatic carcinoma of prostate was made.
\end{abstract}

Keywords: Prostate, cervical lymphadenopathy, PSA.

\section{Introduction}

It is estimated that there are almost 1.3 million new cases of prostate cancer, ranking as the second most frequent cancer and the fifth leading cause of cancer death in men. It is the most frequently diagnosed cancer among men in over one-half (105 of 185) of the countries of the world $^{(1)}$. Early-stage prostate cancer usually has no symptoms; men with more advanced disease may experience genitourinary signs and symptoms. Advanced prostate cancer commonly spreads to the bones, which can cause pain in the hips, spine, ribs, or other areas. The most common metastatic site is the lymph nodes, usually those of the pelvis and retroperitoneum ${ }^{(2)}$. The most frequent non head or neck primary cancers 


\section{JMSCR Vol||07||Issue||06||Page 1068-1072||June}

metastatic to the cervical chain are lung, breast, and kidney ${ }^{[3]}$. Cervical lymphadenopathy is very rare in metastatic carcinoma prostate ${ }^{(4)}$. We report a case with Cervical lymph node metastasis in Carcinoma prostate with no genitourinary symptoms.

\section{Case Report}

A 90-year-old male presented to the outpatient department with pain in neck, enlargement of left side cervical lymph nodes and loss of appetite for 3 months duration. Physical examination revealed left cervical lymph nodes enlargement, $4-5 \mathrm{~cm}$ in size, hard and fixed (Fig1). There was no peripheral lymphadenopathy. Digital rectal examination revealed mildly an enlarged prostate with sulcus obliterated, which was hard in consistency. The patient did not have any urinary complaints. Chest X-ray was normal. FNAC from lymph node revealed metastatic adenocarcinoma (Fig-2). CT Neck and Chest revealed heterogeneously enhanced lymph node mass in left cervical level III, and IV and multiple small lymph nodes in pre and para-aortic region (Fig-3). USG abdomen shows simple cyst in left kidney. Serum PSA was highly raised with value ranging $269 \mathrm{ng} / \mathrm{ml}$. Based on raised Serum PSA value diagnosis of Carcinoma prostate was made. Bilateral subcapsular orchidectomy was performed and patient kept on Bicalutamide 50 mg per oral once daily. Patient was followed up and there was a remarkable response to hormonal therapy, with improved general condition, improved appetite and complete resolution of cervical lymphadenopathy.

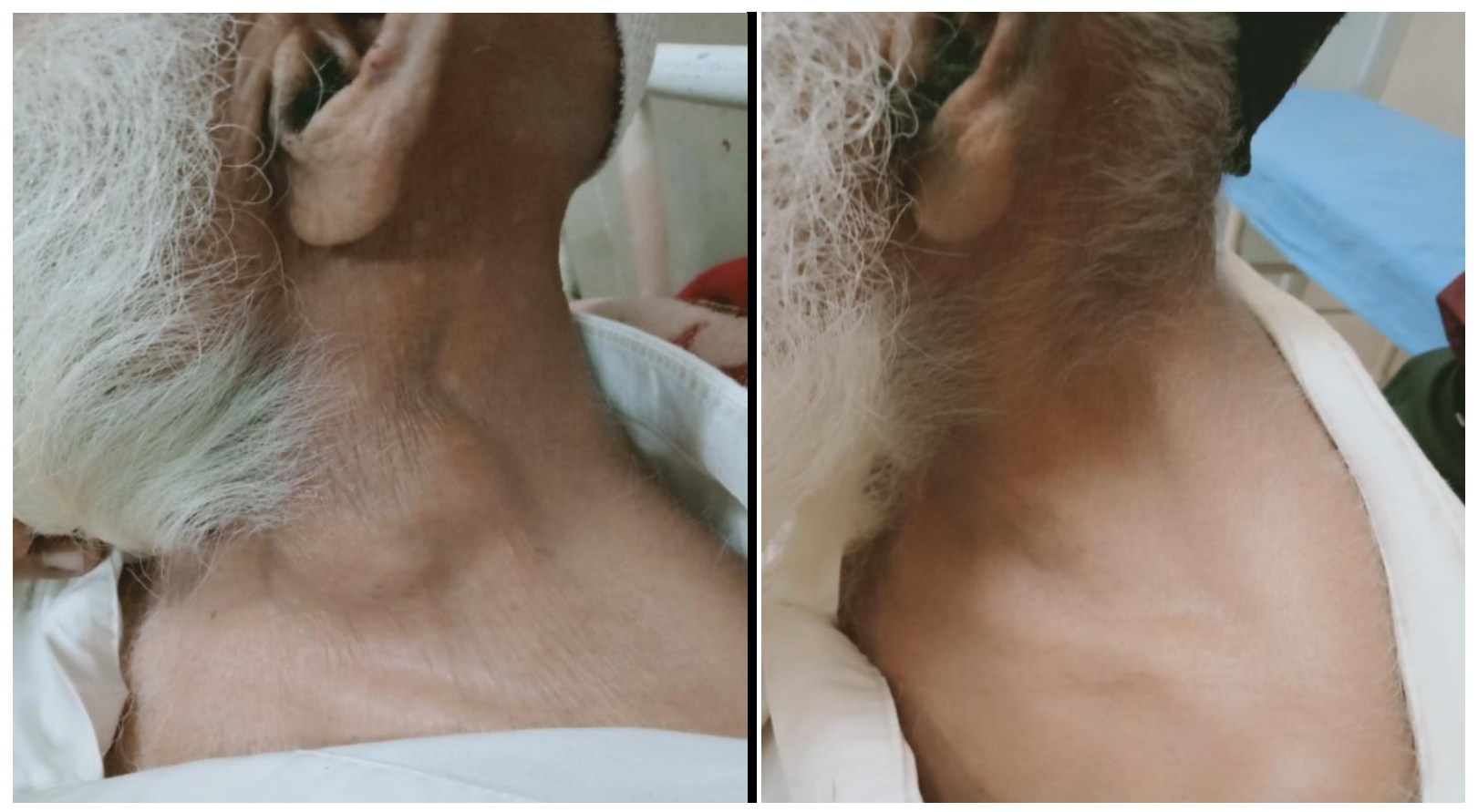

Figure 1

(a) Initial presentation with cervical lymphadenopathy. (b). After treatment. 


\section{JMSCR Vol||07||Issue||06||Page 1068-1072||June}

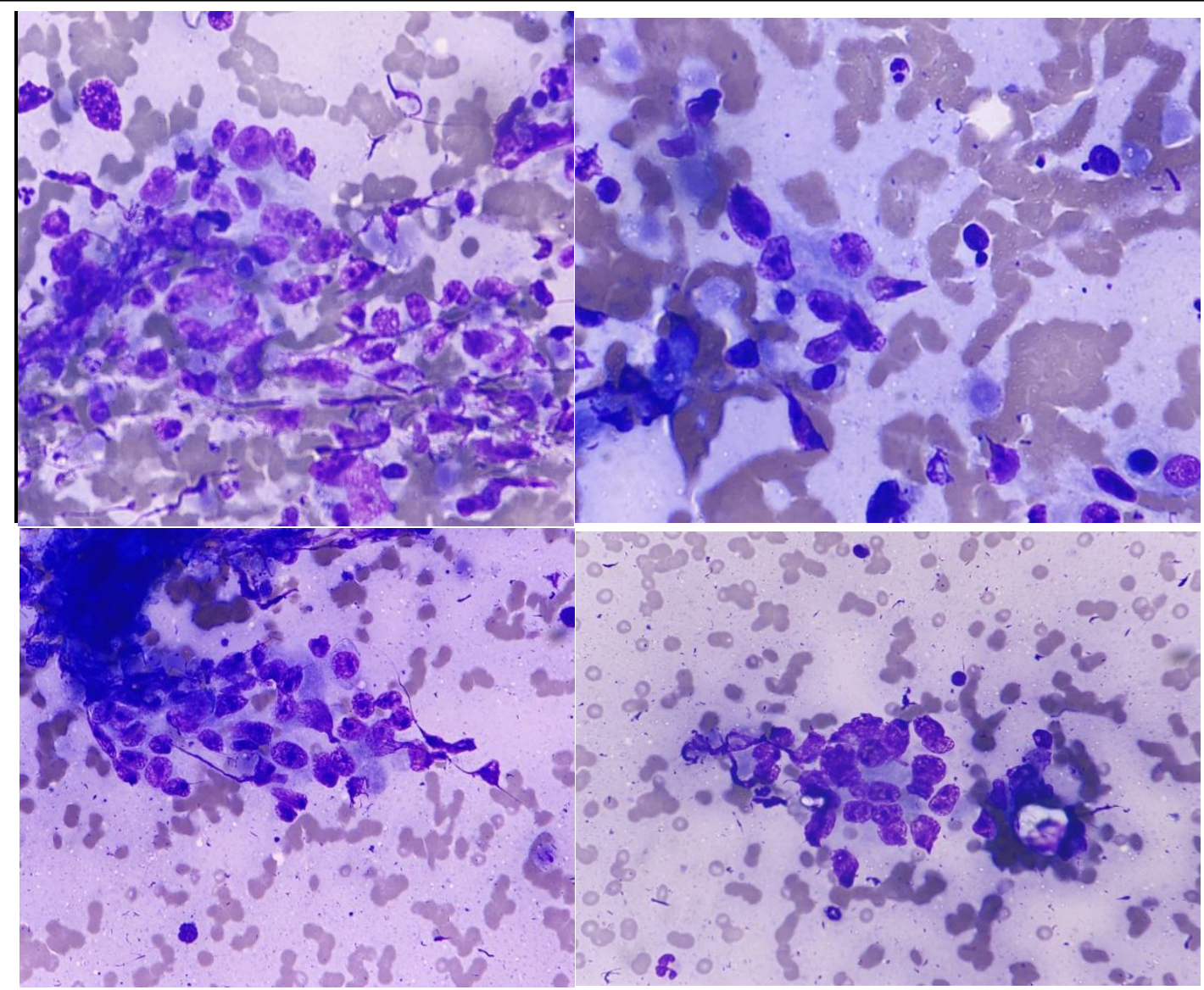

Figure 3

Cytology smears showing pleomorphic epithelial cells arranged in clusters, fragments and acini pattern. The cells show high N:C ratio with fine chromatin, prominent nucleoli and moderate cytoplasm suggestive of adenocarcinoma. (giemsa 10x,40x).
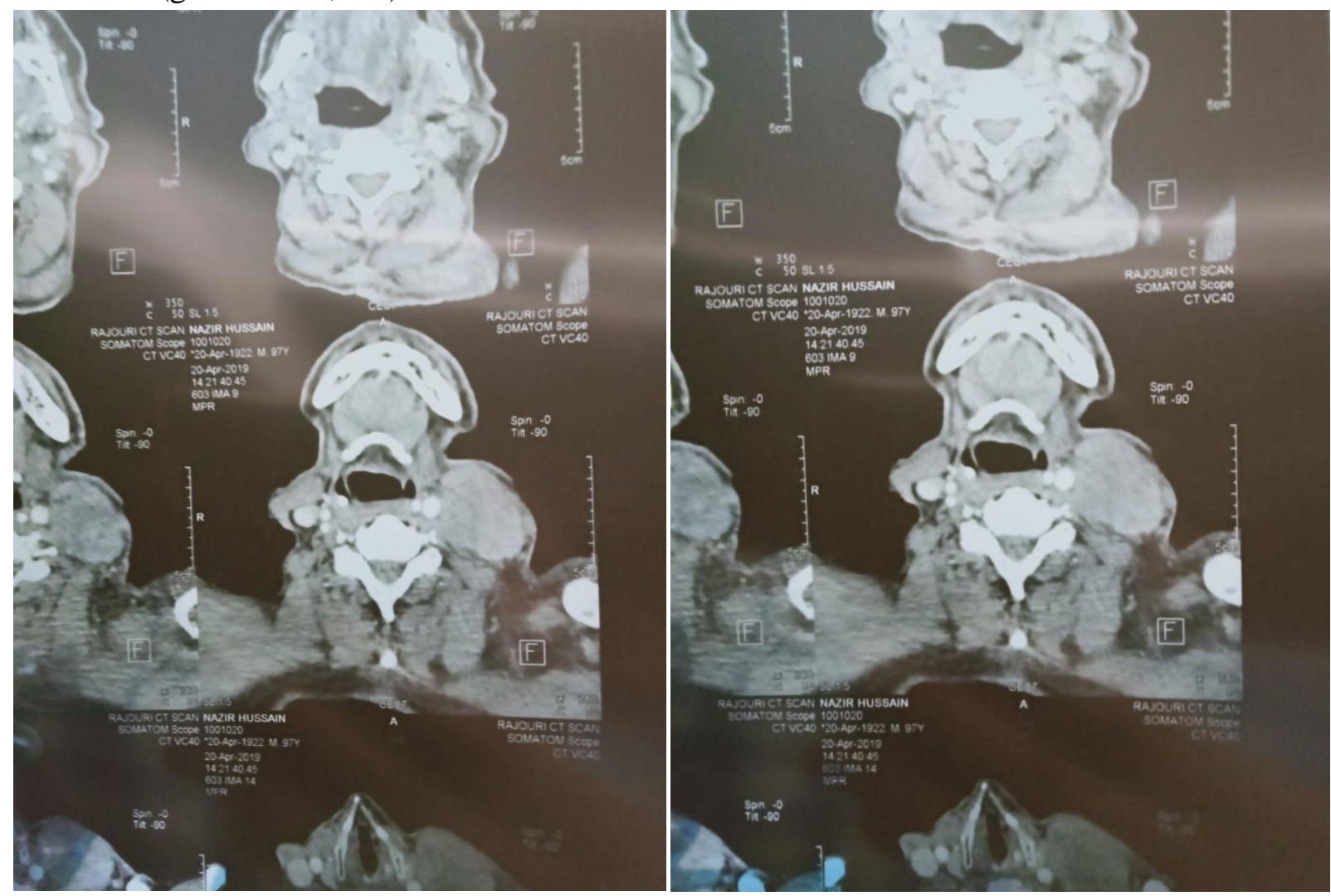

CT Scan of Neck suggestive of left cervical lymphadenopathy $4-5 \mathrm{~cm}$. 


\section{Discussion}

Prostate cancer is the most common cancer in men in many Western countries. Prostate cancer spreads primarily to the regionallymph nodes and bones, followed by lung, bladder, liver, and adrenal gland. Involvement of cervical lymph nodes inprostate cancer is rare and these patients over 45 years usually have widespread metastatic disease. The reported incidence varies between 0.28 and $0.4 \%$ in most series, with only one case in the literature presentingin a man younger than 45 years of age $e^{[5.6]}$. The most common presentation in an asymptomatic patient with an incidental finding on routine digital rectal examination or an elevated PSA, or voiding difficulties. Other presenting symptoms include hematuria, urinary retention, bone pain, and perineal pain ${ }^{[7]}$.

Various hypotheses have been documented in the literature for cervical lymph node metastasis from prostate cancer. First is the hematogenous spread via the vertebral venous system, or Batson's plexus ${ }^{[8]}$ while the other is the lymphatic spread via the lymphatics in the prostate that drain obturator-hypogastric and presacral nodes and from these to the iliac, paraaortic, cisterna chyli, and thoracic duct. Finally, the lymphatic drainage enters the systemic blood circulation via the left subclavian vein. Some authors postulated that tumor cells can lodge in the left cervical nodesby retrograde spread due to the proximity of these nodes with the point-of-entry of the thoracic duct into the left subclavian vein ${ }^{[9]}$. Cho and Epstein identified 26 patients with metastatic prostate cancer in the supradiaphragmatic lymphnodes from 1972 through 1987, 15 of which were in the supraclavical nodes ${ }^{(10)}$. Saitoh et al. found that $0.4 \%$ (5 of 1367 patients) of the patients with prostate cancer at autopsy had metastases to the cervical lymph nodes ${ }^{(11)}$. Cho and Epstein in their study found that $35 \%$ of patients had no evidence of bony metastases, $24 \%$ had normal serumacid phosphatase levels, and $42 \%$ had no prostatic abnormalities on digital rectal exam. Jones and Anthony in their study found that PSA or prostate specific acid phosphatase levels were elevated in only five of nine patients with cervical metastases $^{(12)}$.

Cho KR et al, reported on the prognosis after such a presentation was generally poor, with a mean survival of 19.8 months (range, 1-46 months) ${ }^{(5)}$. Jones and Anthony reported 11 patients with prostate cancer presenting with cervical lymphadenopathy. Six patients survived for 1-101 months (average, 25.8 months) and five died at 766 months (average, 34.4 months) with a combined survival of 29.7 months $^{(12)}$. However, Chitale et al, presented a patient with prostate cancer presenting with cervical lymphadenopathy who received regular hormone therapy and remained symptom-free for 9 years ${ }^{(13)}$.

\section{Conclusion}

Prostate cancer must be considered among the differential diagnosis of men presenting with supraclavicular or any cervical lymphadenopathy, regardless of the age or absence of lower urinary tract symptoms. A thorough physical examination including digital rectal examination, measurement of serum prostate specific antigen, cytology and histopathology along with immunohistochemical stains play an important role in establishing the diagnosis of the prostate cancer in such an unusual presentation.

\section{References}

1. Bray F, Ferlay J, et al. Globocan Cancer Statistics 2018: Globocan Estimates of Incidence and Mortality Worldwide for 36 cancers in 185 Countries. CA: Cancer J Clin. 2018; 0:1-31.

2. Carleton J, P Vander Riet, Dahm P: Metastatic Prostate cancer presenting as an asymptomatic neck mass. Prostate Cancer and Prostatic Diseases, 2005; 8: 293-95

3. M. P. J. Yardley, "Investigation of cervical lymphadenopathy presumed to be metastatic in nature: a review of current clinical practice," Journal of the Royal College of Surgeons of Edinburgh, vol. 37, no. 5, pp. 319-321, 1992. 
4. Platania M, Bajetta E, Guodalupi V et al: Prostate adenocarcinoma presenting with supraclavicular node enlargement: report of a case. Tumori, 2008; 94: 769-71

5. Cho KR, Epstein JI: Metastatic prostatic carcinoma to supradiaphragmatic lymph nodes, a clinicopathologic and immunohistochemical study. Am J Surg Pathol, 1987; 11: 457-63

6. N Eke, MK Sapira: Prostate cancer in Port Harcourt, Nigeria: features and outcome. Nigerian Journal of Surgical Research, 2002; 4(1): 34-44

7. Lin YY, Lin DS, Kang BH, Lin YS. Neck mass as the first presentation of metastatic prostatic adenocarcinoma. J Chin Med Assoc 2011;74:570-73.

8. Batson OV (1995) The function of the vertebral veins and their role in the spread of metastases. Clin Orthop Relat Res 312: 4-9.

9. H. Jones and P. P. Anthony, "Metastatic prostatic carcinoma presenting as leftsided cervical lymphadenopathy: a series of 11 cases," Histopathology, vol. 21, no. 2, pp. 149-154, 1992.

10. Saitoh H, Hida M, Shimbo T, Nakamura K, Yamagata J, Satoh T. Metastatic patterns of prostate cancer: correlation between sites and number of organs involved. Cancer 1984;54:3078-84.

11. Copeland B et al. Prostate carcinoma metastatic to the cervical lymph nodes: report of two cases and review of the literature. Am J Otolaryngol 2001; 22: 420-423.

12. Jones H, Anthony PP. Metastatic prostatic carcinoma presenting as left-sided cervical lymphadenopathy: a series of 11 cases. Histopathology 1992;21:149-54
13. Chitale SV, Harry L, Gaches CGC, Ball RY. Presentatio of prostatic adenocarcinoma with cervical lymphadenopathy: two case reports and review of the literature. Otolaryngol Head Neck Surg 2001;125:431-2. 